\title{
THE ROOTS OF CORONAL STRUCTURE IN THE SUN'S SURFACE
}

\section{LEON GOLUB}

Smithsonian Astrophysical Observatory, Harvard-Smithsonian Center for Astrophysics, Cambridge, MA 02138, U.S.A.

and

\section{HAROLD ZIRIN and HAIMIN WANG}

Big Bear Solar Observatory, California Institute of Technology, Pasadena, CA 91125, U. S.A.

(Received 7 July, 1993; in revised form 13 April, 1994)

\begin{abstract}
We have compared the structures seen on X-ray images obtained by a flight of the NIXT sounding rocket payload on July 11, 1991 with near-simultaneous photospheric and chromospheric structures and magnetic fields observed at Big Bear. The X-ray images reflect emission of both $\mathrm{Mg} \mathrm{X}$ and $\mathrm{Fe} \mathrm{XVI,} \mathrm{formed} \mathrm{at} 1 \times 10^{6} \mathrm{~K}$ and $3 \times 10^{6} \mathrm{~K}$, respectively. The brightest $\mathrm{H} \alpha$ sources correspond to a dying sub-flare and other active region components, all of which reveal coronal enhancements situated spatially well above the $\mathrm{H} \alpha$ emission. The largest set of X-ray arches connected plages of opposite polarity in a large bipolar active region. The arches appear to lie in a small range of angle in the meridian plane connecting their footpoints. Sunspots are dark on the surface and in the corona. For the first time we see an emerging flux region in X-rays and find the emission extends twice as high as the $\mathrm{H} \alpha$ arches. Many features which we believe to correspond to 'X-ray bright points' (XBPs) were observed. Whether by resolution or spectral band, the number detected greatly exceeds that from previous work. All of the brighter XBPs correspond to bipolar $\mathrm{H} \alpha$ features, while unipolar $\mathrm{H} \alpha$ bright points are the base of more diffuse comet-like coronal arches, generally vertical. These diverge from individual features by less than $30^{\circ}$, and give a good measure of what the 'canopies' must do. The $\mathrm{H} \alpha$ data shows that all the $\mathrm{H} \alpha$ features were present the entire day, so they are not clearly disappearing or reappearing. We find a new class of XBPs which we call 'satellite points', elements of opposite polarity linked to nearby umbrae by invisible field lines. The satellite points change rapidly in X-ray brightness during the flight. An M1.9 flare occurred four hours after the flight; examination of the pre-flare structures reveals nothing unusual.
\end{abstract}

\section{Introduction}

From the early days of coronal observations, it was clear that there is an association between the intensity and structure of the corona and the pattern of active regions on the Sun's surface. With the advent of direct imaging of the corona on the disk from sounding rockets (Vaiana, Krieger, and Timothy, 1973) and from Skylab (Vaiana et al., 1975), that relationship became more clear. At high resolution (Webb and Zirin, 1981), one would find fairly good correlation between coronal structures and those seen in magnetograms and monochromatic chromospheric images, from coronal holes to the smallest X-ray bright points, which could be identified with bright $\mathrm{H} \alpha$ features and with He 10830 'dark points' (Harvey et al., 1975).

With the advent of normal incidence imaging of the corona (Golub et al., 1990; Walker et al., 1988; Underwood et al., 1987), a new level of resolution in the $\mathrm{X}$-ray images could be obtained. To date, no systematic comparison between the 
new coronal data and the lower levels of the atmosphere has been carried out. The purpose of this work is therefore to study the association between the coronal structures and the underlying magnetic fields which produce them. Insofar as the heating and origin of the corona are still a mystery, it is important to understand the relation of the coronal emission measure with the underlying surface morphology. It is taken as a given that the coronal structure results from the underlying magnetic structure, since no magnetic poles have ever been observed in the corona and the local pressure would be inadequate to support such fields.

\section{The Data}

The data basis for the present work is a rocket flight which took place at the time of the total eclipse of July 11, 1991 (17:28-17:32 UT). We compare the images obtained by that rocket at 17:30 UT with a number of observations made at the Big Bear Solar Observatory in support of eclipse observations with optical and radio telescopes. Because the BBSO observations were directed at eclipse support, the data obtained were less comprehensive than we would have wished for this study, but nonetheless offered considerable chance for comparison. Our studies were conducted by comparing both the large- and small-scale structures on magnified and enhanced magnetogram and $\mathrm{H} \alpha$ images.

What made the rocket observations possible was the extraordinary coincidence that first contast over White Sands, New Mexico did not occur until just after third contast as seen from Hawaii; thus, we were able to observe the unocculted on-disk corona simultaneous with Hawaii's totality, and the rocket pointing system was not rendered dysfunctional by having to deal with a non-circular solar disk.

The general comparison between the coronal observations and $\mathrm{H} \alpha$ is easily seen from Figures 1 and 2, which display successively the simultaneous (within seconds) full-disk $\mathrm{H} \alpha$ image and the full-disk X-ray image. The NOAA numbers for active regions are given on the figures, along with ID numbers or letters for other features. The high-resolution images cover typically $240 \times 300 \mathrm{arc} \mathrm{sec}$, and we have matched the scales of the different wavelengths as closely as possible, following the orientation $\mathrm{N}$ top, W right. It is immediately clear to the observer that there is a good correspondence between all bright $\mathrm{H} \alpha$ structures and bright areas in X-rays. It is also clear that the dark filaments on the disk correspond to regions where the X-ray emission is absent. Furthermore, there is a considerable X-ray structure connecting the bright regions that we know to be magnetic poles. Within that range, there is a good deal of variation of the relative intensity of $\mathrm{X}$-rays and $\mathrm{H} \alpha$, which we shall investigate.

The X-ray images were taken in a $1.4 \AA$ bandpass at $63.5 \AA$ (Golub et al., 1990) and combine the intensities of two coronal lines, one of $\mathrm{Mg} \mathrm{X}$, which arises from plasma at about one million degrees, and one of Fe XVI, which is produced at three million degrees. These wavelengths were chosen for recording the temperatures associated with the quiet corona and with active regions, respectively; the NIXT 


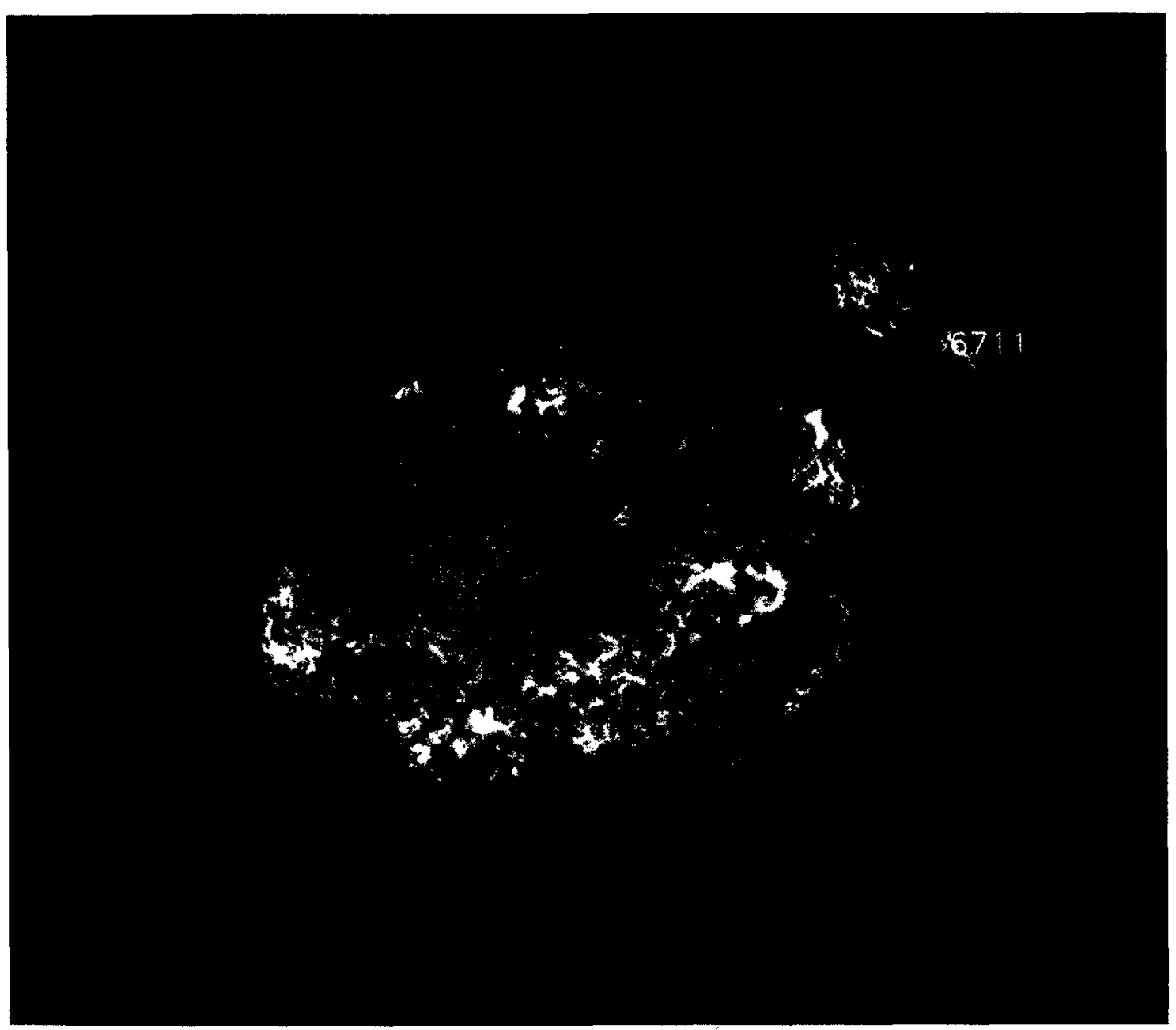

Fig. 1. Full-disk $\mathrm{H} \alpha$ taken at 17:30:50 at the time of the eclipse.

temperature response is discussed in Golub and Herant (1989). With a single passband we have no way other than by inference from the structure to determine the relative contribution of these lines, or whether temperature or emission measure variations determine brightness variation in any particular region.

The $\mathrm{MgX}$ response of the NIXT telescope is also responsible for the often striking correspondence between chromospheric and $\mathrm{X}$-ray brightening. This has been explained by constructing hydrostatic models of coronal loops and folding the NIXT instrument response through the calculated emission distributions (Peres, Reale, and Golub, 1991). Assuming that a given loop has a coronal portion which reaches a temperature of several million degrees, it is found that there is a sharp peak in the NIXT brightness near the base of the loop as its temperature falls through one million degrees, where the $\mathrm{Mg} \mathrm{X}$ peak occurs. This cool region is thin but dense, and reflects the enhanced density overlying chromospheric regions of high magnetic field strength. The models also clearly show that these footpoint brightenings only occur for certain values of the loop parameters, usually for loops with high pressures such as are found in active regions. The $\mathrm{Mg}$ X regions can easily 


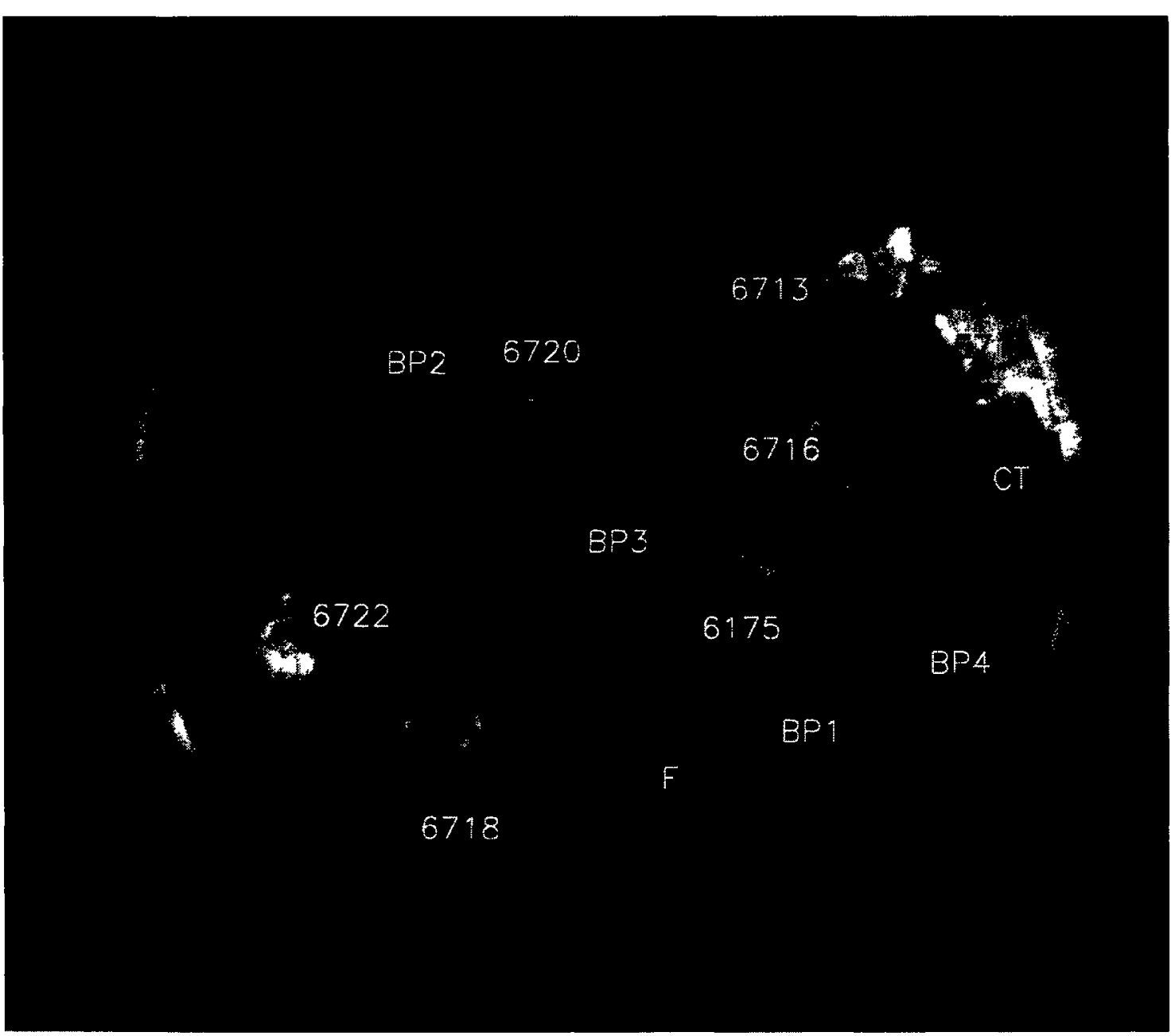

Fig. 2. NIXT X-ray image corresponding to Figure 1; exposure duration is $30 \mathrm{~s}$, starting at 17:28:16 UT.

be recognized by a lack of vertical structure and by their close correspondence to $\mathrm{H} \alpha$ plage.

In the next section we compare the detailed structure region by region. Please note that while the $\mathrm{H} \alpha$ and X-ray full-disk frames are nearly simultaneous, the high-resolution BBSO frames were taken at other times (which may be read from the clock on the $\mathrm{H} \alpha$ frame), so there are some differences in transient features, particularly in region 6713. In each case the data on individual regions consists of $\mathrm{H} \alpha$ images and longitudinal magnetograms, which are displayed to the same scale. The calibration of the magnetograph is given by Varsik (1994).

\section{Individual Regions}

NOAA 6713

We begin our discussion with NOAA 6713 (Figure 3(a-c), respectively X-ray, VMG, $\mathrm{H} \alpha$ ), which is particularly interesting because it is in a relatively high state of activity. In the center of Figure 3(a) we see a sunspot $(S)$ with a filament $(F)$ 


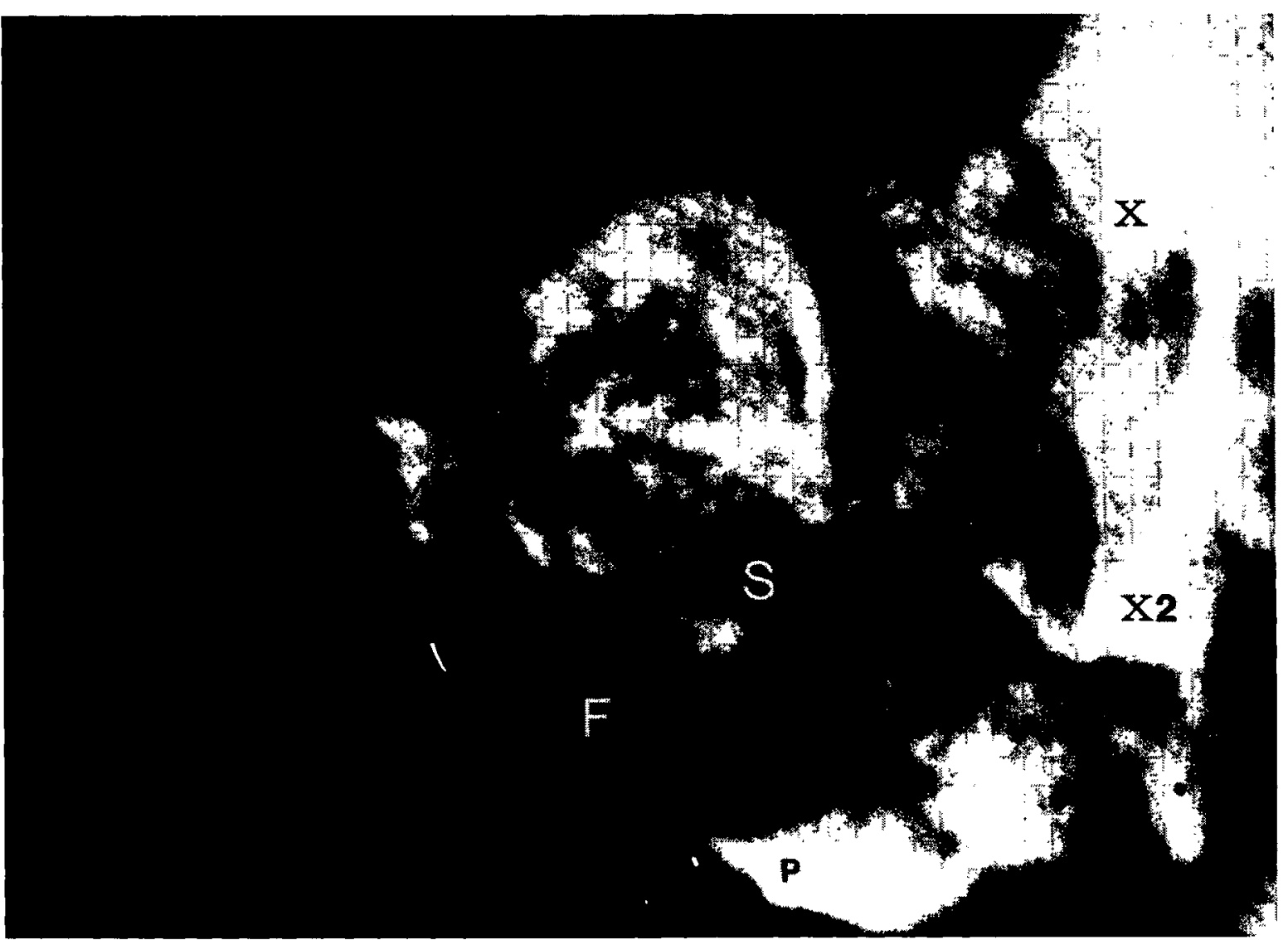

Fig. 3a.

Fig. 3. (a) X-ray, (b) VMG, and (c) $\mathrm{H} \alpha$ images of the region 6713. These images are in matching scale as close as possible, and roughly $5 \times 4$ arc min. In this and all following figures, north is up and west is to the right. This and subsequent X-ray images are portions of the full frame shown in Figure 2.

looping around it. The filament is dark in the X-ray photo. From the plage $(P)$ just this side of the filament, a bundle of coronal loops appears to arch across the filament filling in the absorption at right. The spot itself is dark, but bordered by emitting material. The brightest feature in the $\mathrm{X}$-ray data is lcoated at $X$, where a small subflare occurred just before the flight.

An arch filament system (AFS) at $E$ is the telltale $\mathrm{H} \alpha$ indication of the presence of an emerging flux region, or EFR. This emergence was confirmed on the next day by the appearance of a mature bipolar region with an umbra that had grown larger than the main umbra in this picture. EFRs have been observed before (Webb and Zirin, 1981) and invariably are bright in both X-rays and $\mathrm{H} \alpha$. They represent new magnetic flux loops pushing up into the previously existing magnetic fields, and presumably the heating due to the reconnection and restructing of the magnetic field produces the high brightness.

This is the first case in which the vertical extent of the coronal portion of the emerging dipole has been observed. The height of the cap of hot coronal material in the EFR is approximately twice that of the $\mathrm{H} \alpha \mathrm{AFS}$, about $45000 \mathrm{~km} . \mathrm{H} \alpha$ movies 


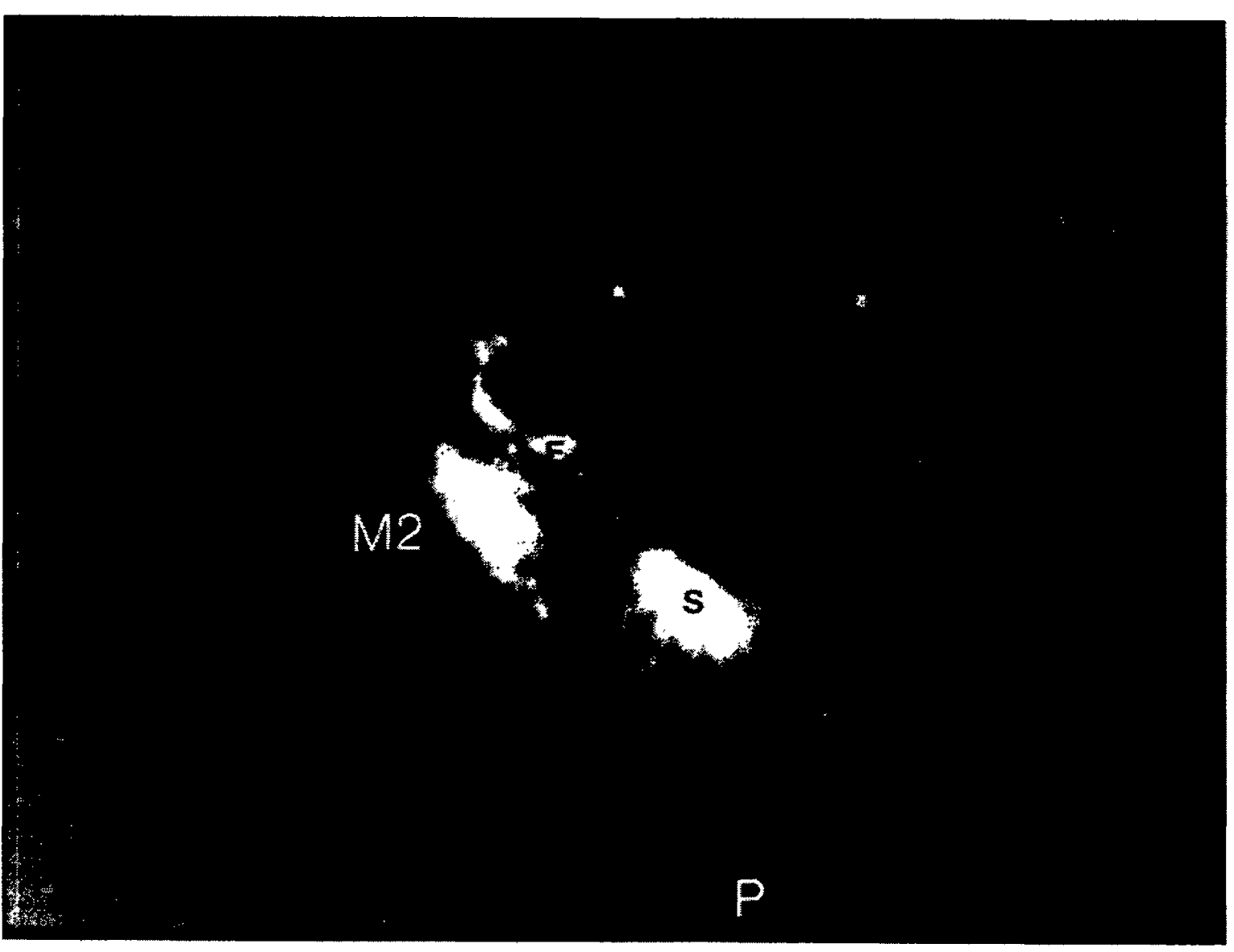

Fig. 3b.

of these loop systems show them (Chou and Zirin, 1988) to rise steadily and break up at the top of the $\mathrm{H} \alpha$. The EFR, while bright, is less bright than the remnant of subflare $X$, and shows dark arches which appear to match the AFS. The bright $\mathrm{X}$-ray loops begin just above the AFS, and thus in some sense precede the AFS into the corona as the EFR rises.

The rather small flare at $X$ produces the brightest feature in the $\mathrm{X}$-ray photo, with a bright coronal cloud considerably larger than the $\mathrm{H} \alpha$ flare, about $50000 \mathrm{~km}$. It was detected on the BBSO full-disk $\mathrm{H} \alpha$ patrol just prior to the NIXT flight, and by the GOES satellite (peak emission C3.7). We note, however, that a surge in the northern prior of AR 6713 during the time of the X-ray observations produced no detactable X-ray signature (Schmieder et al., 1992). The flare at $X$ occurred in a completely unipolar plage; so we assume that the $\mathrm{H} \alpha$ flare consists of only surface footpoints of a fairly extensive coronal cloud. This is confirmed by an elegant loop in the X-ray image that arches over the top from the flare to the point X2. There is brightening in region 6711 in an area at the end of the filament, which may also have been connected to the flare. Between the EFR and the subflare is an area with several X-ray structures that are anomalously bright; this is because of bright material projecting from foreground plages. 


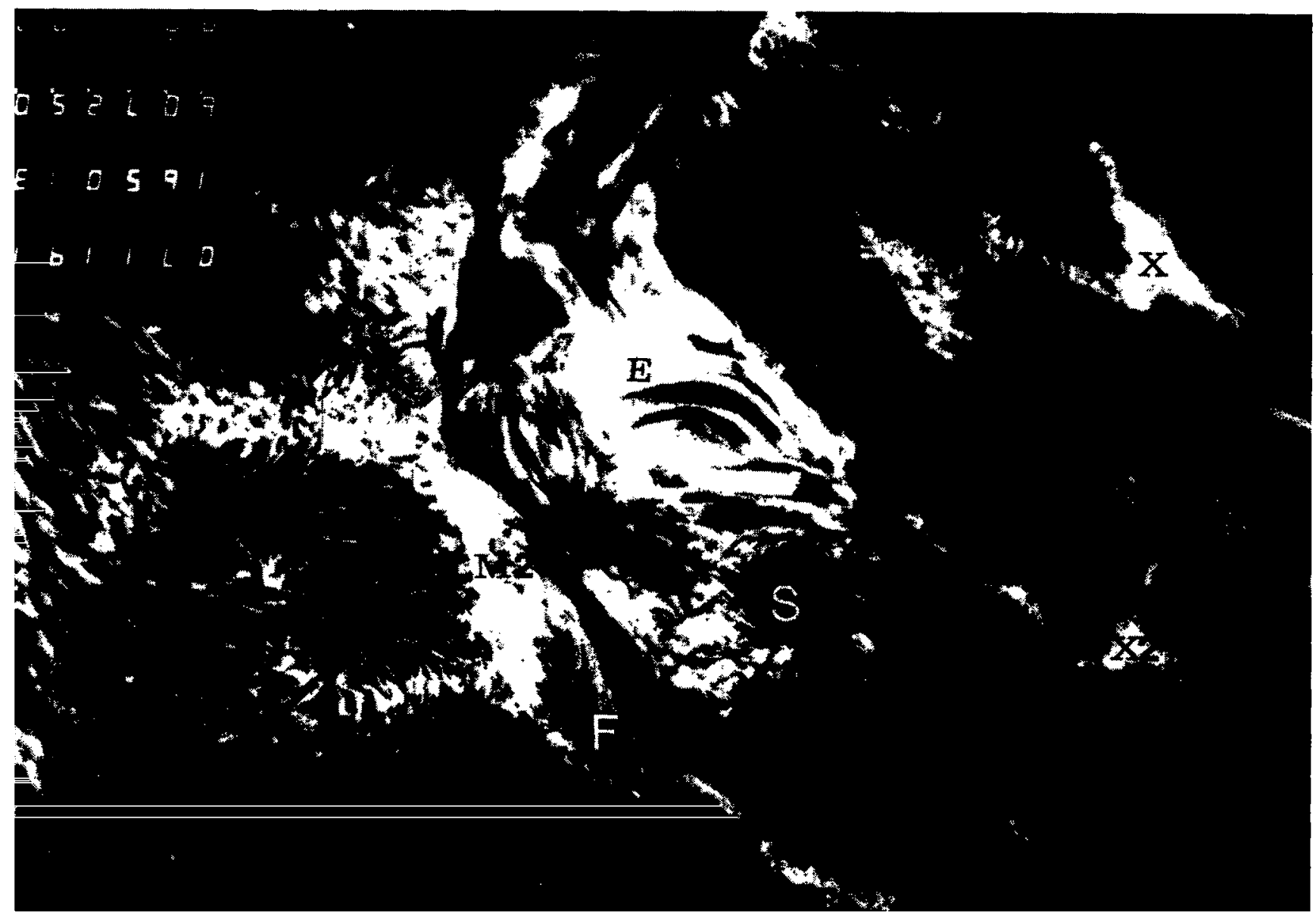

Fig. 3c.

About four hours after the eclipse (21:31 UT), a two-ribbon M1.9 flare occurred at point M2, but the filament did not erupt. Thus we have an excellent view of the pre-flare state in X-ray. This is of some interest in flare models; in fact there is nothing obviously different in this area that tells us a substantial flare is about to occur. It is interesting that X-ray emission loops crossed the end of the filament that erupted, and there have been suggestions that loop crossing is a flare precursor.

While the absence of $\mathrm{X}$-ray emission from filaments has generally been interpreted as due to a lack of overlying corona, in several cases on our images we clearly see the filament blocking background emission. This means that the optical depth in the Lyman continua of $\mathrm{H}$ or $\mathrm{He}$ is near unity (there is little $\mathrm{He}$ II in filaments) and defines a minimum amount of neutral hydrogen or helium along the path through the filament. At the NIXT wavelength, the opacity will be dominated by Lyman-continuum absorption of neutral $\mathrm{H}$ and $\mathrm{He}$. Using the values in Allen $(1981$, p. 98), we calculate the neutral hydrogen photoionization cross section at the NIXT wavelength to be $\alpha_{\nu}=2.7 \times 10^{-21} \mathrm{~cm}^{2}$, and that for He to 
be $2.8 \times 10^{-20} \mathrm{~cm}^{2}$. Assuming both $\mathrm{H}$ and $\mathrm{He}$ to be largely unionized and in the ground state, a minimum of $4 \times 10^{20}$ neutral hydrogen or $4 \times 10^{19} \mathrm{He}$ atoms is required. This is at the upper end (say $N_{\mathrm{H}}=10^{11}, L=10^{9}$ ) of our picture of filament densities and path length, but still reasonable.

To the right (SW) of this active region is the irregular region, NOAA 6711, which appears to be the remnants of several order regions. Since it is close to the limb, we see various arches and loops in the overlying corona. At the limb we can see several low prominences silhouetted against coronal emission from behind the limb, demonstrating the opacity of filaments at this wavelength. As discussed in the preceding paragraph, filament material is absorbing enough to be seen in the X-ray images at $63.5 \AA$. This is typically not the case at the $10-20 \AA$ wavelengths of traditional grazing-incidence $\mathrm{X}$-ray telescopes, where Lyman continuum opacity is much lower. At $C T$ (covered by the Moon in the $\mathrm{H} \alpha$ ) is a small plage that is crossed by a 'comet-tail' emanating from a foreground plage. These coronal features are straight and well collimated, and almost certainly represent the field lines diverging from individual monopolar magnetic elements. They diverge by no more than $30^{\circ}$, much less than the angle assumed by many authors. Similarly, the group of small unipolar features at the lower right (SW) all have essentially vertical and collimated 'comet tails', which are almost certainly the footpoints of long loops whose brightness falls off because of the decrease of density with height. Near the limb are several $\mathrm{H} \alpha$ plages which correspond to bright $\mathrm{X}$-ray features with arches above.

\section{LOOPS IN NOAA 6718}

NOAA 6718 is an extended region which Gaizauskas (1992) pointed out to be the confluence of two dipoles. It presents a spectacular set of coronal loops extending between the following and preceding spot areas. However, the arches do not in fact connect the big double spots, but rather join nearby plage areas marked $\mathrm{E}$ and W. If we correct for position, the loops lie roughly in a vertical plane connecting these sources. Figures 4(a) and 4(b) show enlarged X-ray and magnetic images of this region, and Figure 4(c) shows the $\mathrm{H} \alpha$ image. The leader spots are labeled $P$. The loops clearly arise in $\mathrm{W}$, although a few small arches come from the plage $B$. The umbrae themselves are dark and no bright loops originate there. The arches bypass the follower spots to end in the plage $E$. We cannot at present say why the coronal arches here are so much better populated than those elsewhere. We believe the X-ray brightening which matches the footpoint plages is due to $\mathrm{Mg} \mathrm{X}$ and the loops are from Fe XVI.

The follower spot has three plages near it, of which the brightest is $\mathrm{C}$, with bright $\mathrm{H} \alpha$ and $\mathrm{X}$-ray structure showing a core-halo appearance. $\mathrm{In} \mathrm{H} \alpha$ it is seen to be bipolar, with a small dark filament dividing it. It is also amorphous in $\mathrm{H} \alpha$ rather than granular, as most of the bright $\mathrm{X}$-ray features. That form is characteristic for particularly hot or active $\mathrm{H} \alpha$ plage, and appears to be a measure of the spatial density of hot coronal loops. Bipolar regions are brighter than unipolar ones, 


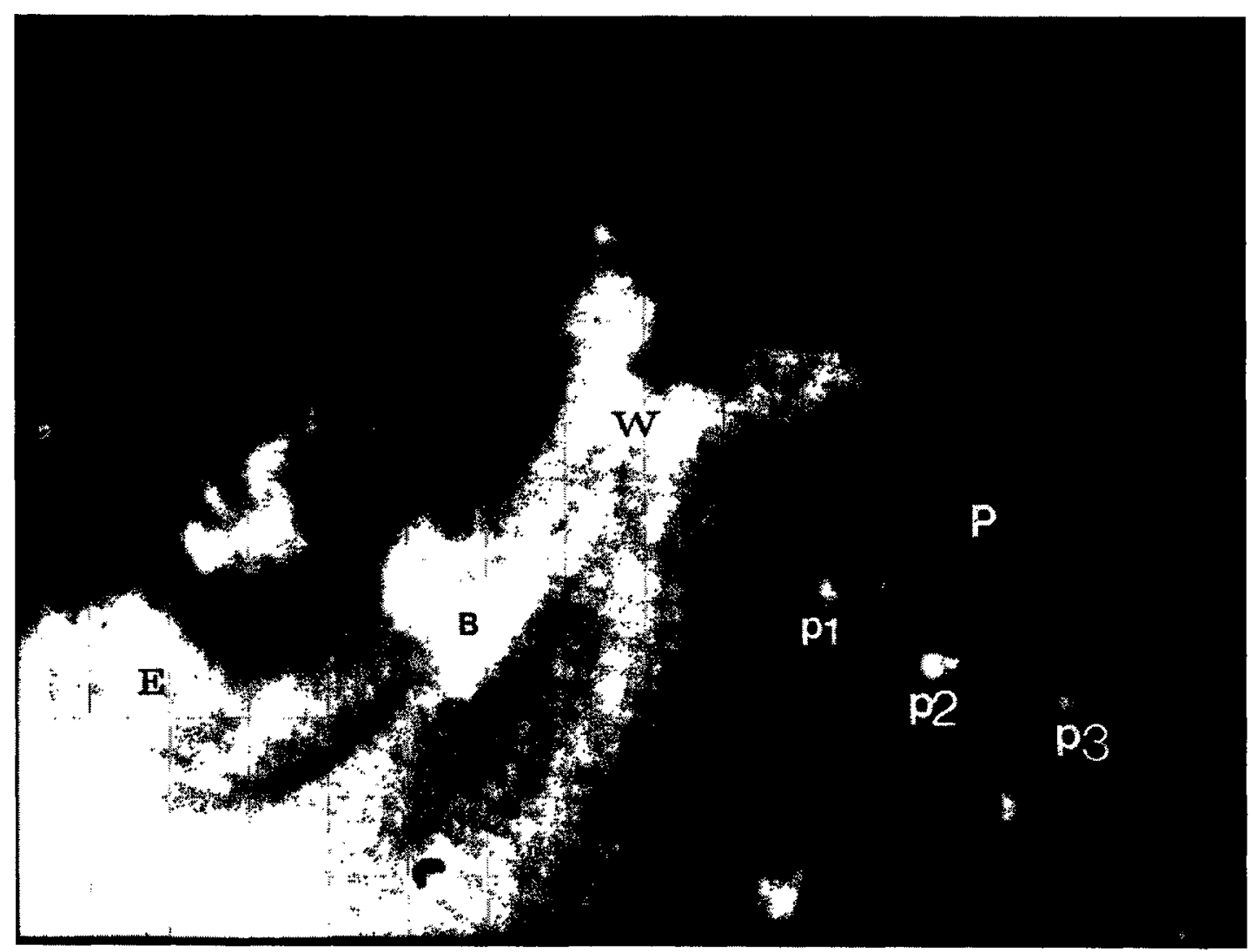

Fig. 4a.

Fig. 4. Matching (a) X-ray, (b) VMG, and (c) H $\alpha$ images of 6718.

because coronal material is trapped in the closed field lines, but the amorphous $\mathrm{H} \alpha$ shows that there is heating besides.

Below the leading umbra of 6718 (marked $P$ ) there are structures which we have not seen before in X-ray images. They consist of three discrete bright points and two more a little further away. Figure 4(b) shows them to be little elements ( $p 1-3$, but they are not $p$ polarity) of satellite polarity (i.e., opposite the main umbra), with two more similar points farther out. These points are much brighter than X-ray bright points (XBPs), which are about the same size, but are usually far away from active regions; to distinguish these we will call them 'Satellite Points'. They are the most rapidly varying features anywhere on the disk in the X-ray data, with two of the satellite points bright on the first frame and fading in the few minutes at altitude (Figure 5). This cooling time scale implies electron densities higher than $10^{10}$. Both $P 2$ and $P 3$ are connected to the main umbra by $\mathrm{H} \alpha$ fibrils, indicating magnetic connection, while $P 1$ is bounded on one side by a filament.

The bright satellite points also are associated with a complicated coronal structure, consisting of several arches extending radially outward from the spot umbra. 


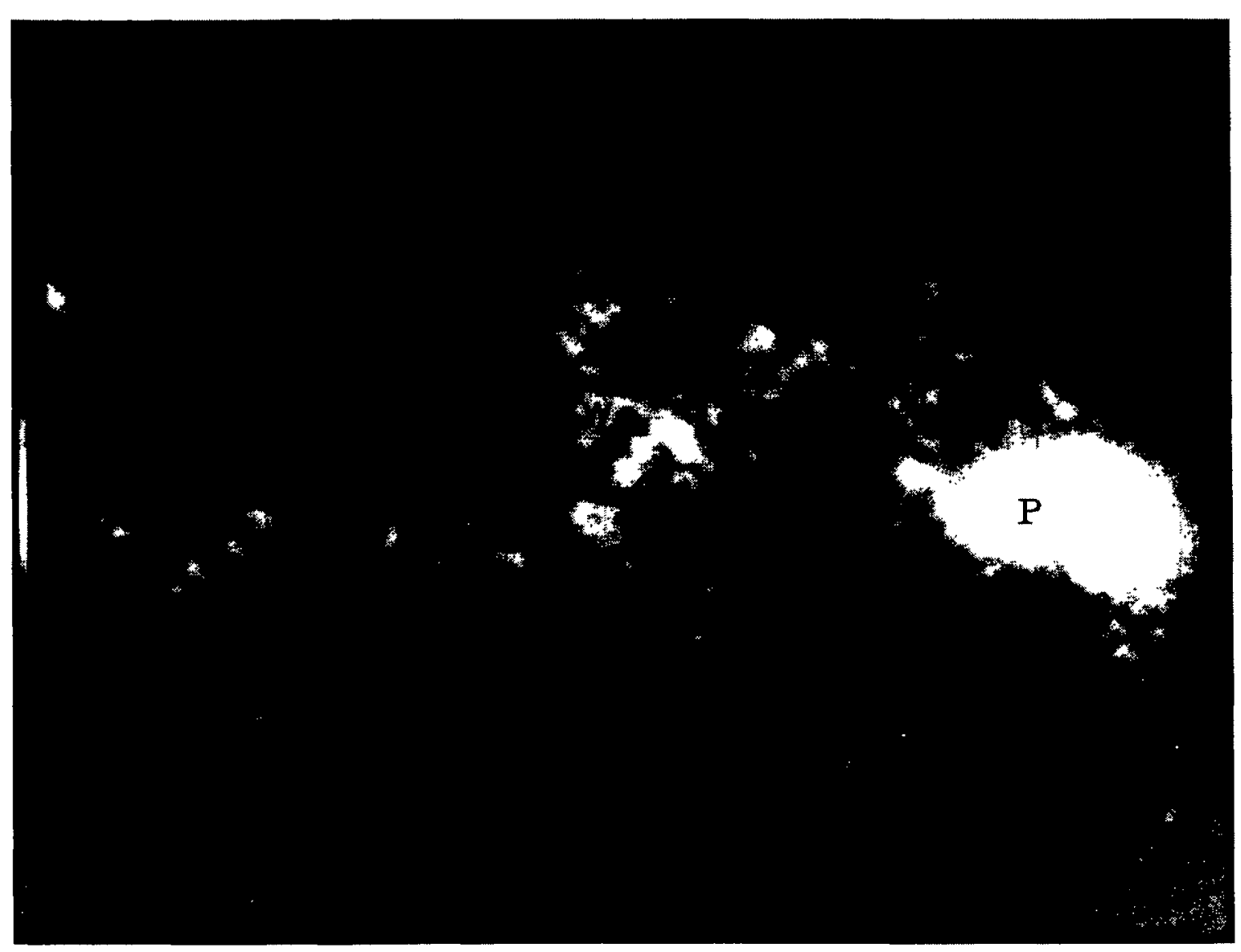

Fig. 4b.

The points are negative unipolar elements, and variations occur in various portions of the radial structure independently. The independent changes along a radial direction imply that there is a serpentine structure, re-entrant to the solar surface several times along the path from spot umbra to satellite point. The relation, if any, to the outflowing 'moving magnetic features' in the moat around the spots is unclear. In $\mathrm{H} \alpha$ we see fibrils passing over the bright points on their way to other opposite polarity features.

Unfortunately the BBSO high-resolution telescopes followed the lunar limb (which is just off the $\mathrm{W}$ limb in Figure 2) during the eclipse, and other highresolution frames were targeted on active regions, so we have little high-resolution $\mathrm{H} \alpha$ or magnetograph data on the XBPs. Our full-disk data show the associated $\mathrm{H} \alpha$ structure of the bright points and whether or not they disappear, but have insufficient resolution to see changes.

Note that although the two plage regions $\mathrm{E}$ and $\mathrm{W}$ that are the base of the large loops do have some $\mathrm{H} \alpha$ structures directed toward one another, there is little in the ground-based data to suggest that these plages are connected by a large coronal loop system. The biggest dark fibrils come out of the follower spot and in fact are dark in X-rays. Even though we expect bright X-rays to overly the active regions, the photospheric observations by themselves do not always indicate the overlying 


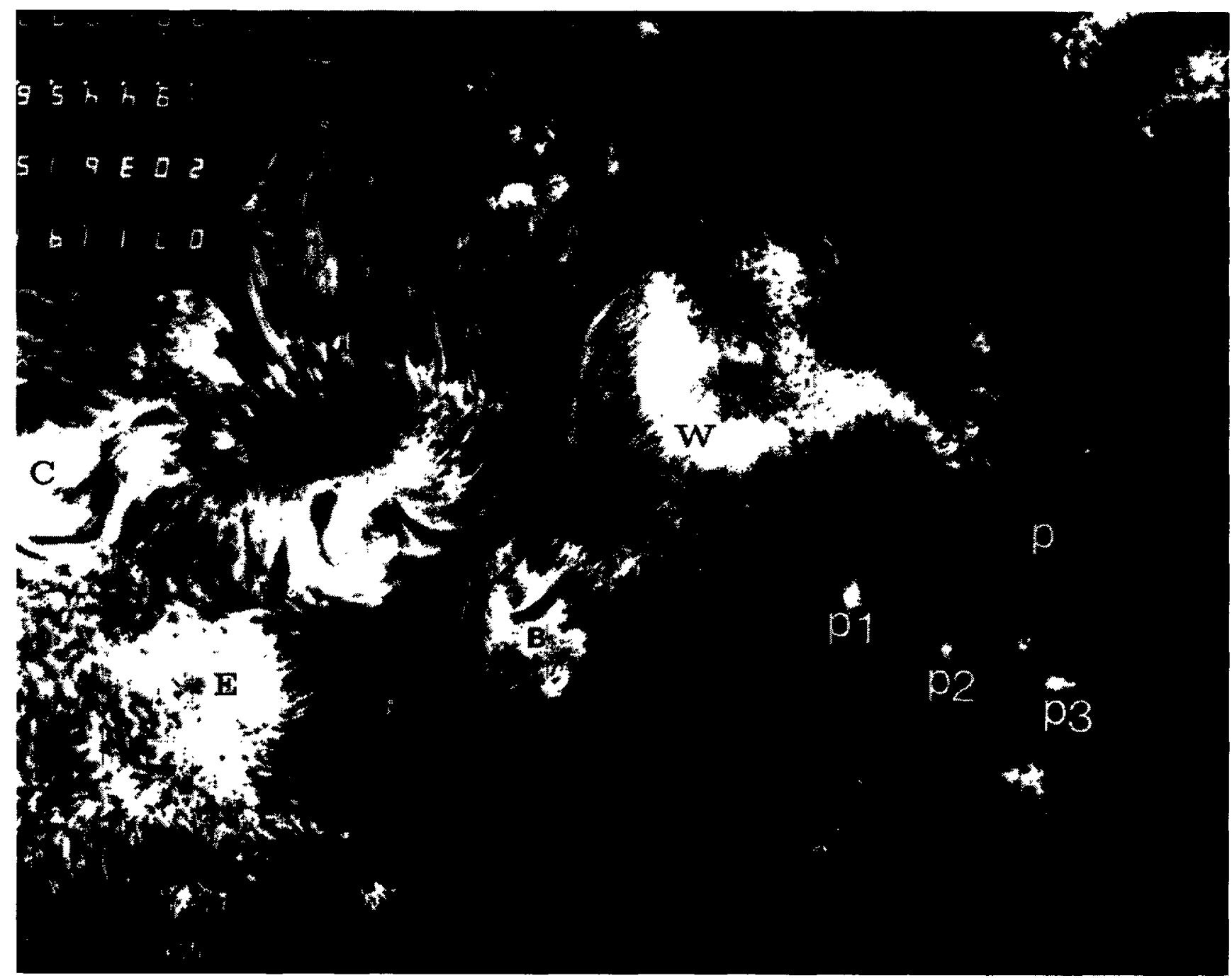

Fig. 4c.

morphology (although this study should help us do so in the future). Why does region 6718 have such big arches? Perhaps because the two regions were joined recently and there is some growth involved. Comparison of the images gives us an interesting glimpse of what the three-dimensional structure in such an active region might be. While the simple EFR in region 6713 seems to have a symmetric hemispherical bright cloud above it, the big arches in 6718 appear to lie in planes radiating from the line of the footpoints.

\section{OTHER REGIONS}

Region 6716 is a classic $\alpha p$ spot group; Figure 6 shows X-ray, VMG, and $\mathrm{H} \alpha$ frames of the region. The dominant $p$ spot is dark, but has a nice set of coronal arches along the filament axis, rather than directly across the neutral line. Particularly striking is the bundle of $\mathrm{X}$-ray arches diverging from the spot $P$, which reminds us that the dominant loops are in fact invisible, cool ones radiating from the umbrae, only 


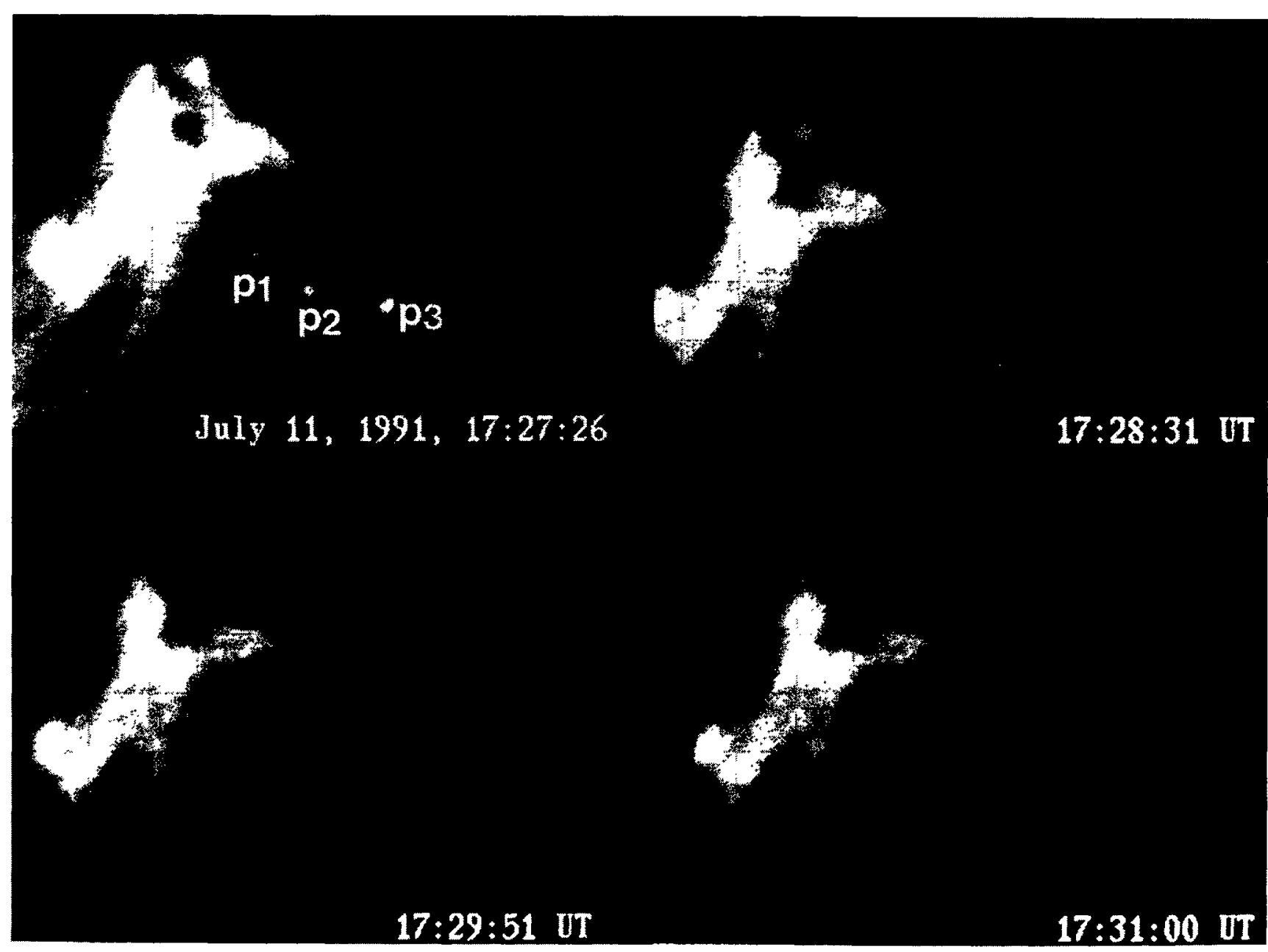

Fig. 5. Four frames showing the rapid variations of the bright points while the rocket was at altitude.

occasionally illuminated by activity. In this region, too, we can see the relation between amorphous plage ( $A$ and $B$ ) and bright $\mathrm{X}$-ray emission. At the $\mathrm{S}$ end of the region is particularly bright plage $(C)$, which is probably bright by the same mechanism as the satellite bright points; it is opposite in polarity to the main spot. Thus, though the umbrae may be dark in X-rays, the other end of their flux loops may be bright.

The correspondence of the dark area $F$ and the $\mathrm{H} \alpha$ filament illustrates the difficulty of distinguishing an area that is dark because there is no longitudinal field there from one absorbed by a filament. Here they cannot be distinguished; the filament fills the longitudinal field-free area on the magnetogram. However, as noted in Section 5, nearer the limb we can detect absorption by the filaments.

Region 6715 is a classic bipolar old plage unlike to produce much excitement. The X-ray image reveals a fine set of loops connecting the preceding and following plages and the leader plage is the source of arches pointing toward more distant places. These are two small filaments which form a ' $V$ ' pointing $\mathrm{W}$ between the two poles, and this is echoed by dark lines in the X-rays. Every bit of the $\mathrm{H} \alpha$ plage is connected to the loops connecting the two poles. Although the coronal 


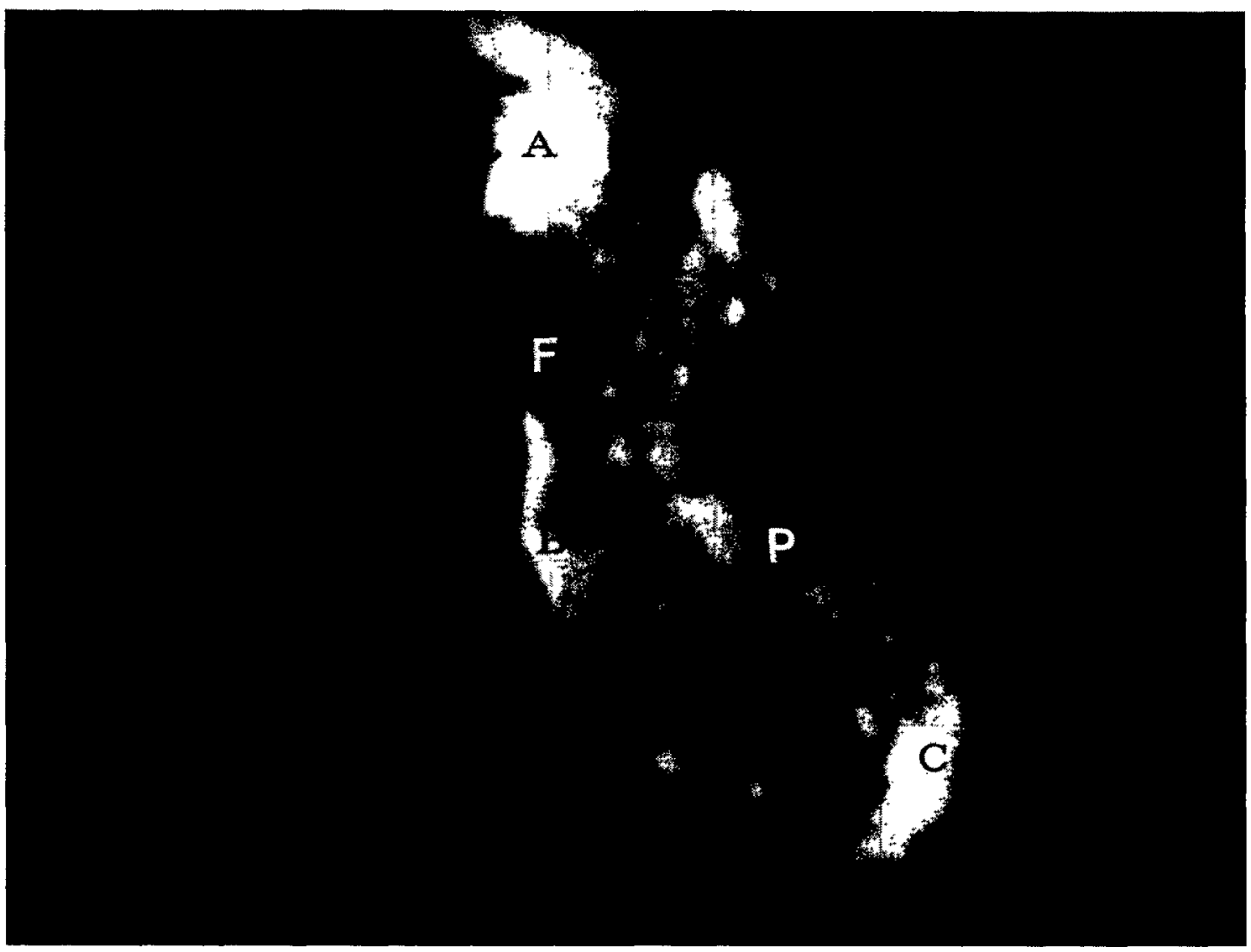

Fig. 6a.

Fig. 6. Matching (a) X-ray, (b) VMG, and (c) $\mathrm{H} \alpha$ images of 6716.

appearance of this active region is very close to what one would extrapolate from a simple dipole potential field, the preceding edge of the region was the location of a series of repeated surges during the ten days preceding the day of the eclipse (Gaizauskas, 1992).

Limbward (SW) of this region, a number of small bright patches of emission are seen, parts of an old and extensive unipolar region. We see that each of these areas is marked by a comet-like structure which we may interpret as a diverging coronal arch or canopy connected to some distant place, in this case mostly to region 6716 . The divergence angle of these comet-like sources varies somewhat, from about $20^{\circ}$ to $60^{\circ}$ in the different regions. They are much more collimated than suggested by various authors (e.g., Giovanelli and Jones, 1982), probably because the $\mathrm{H} \alpha$ spicules generally studied tend to be in more bipolar structures. Presumably this is connected with the exact three-dimensional structure of the magnetic arch configuration. 


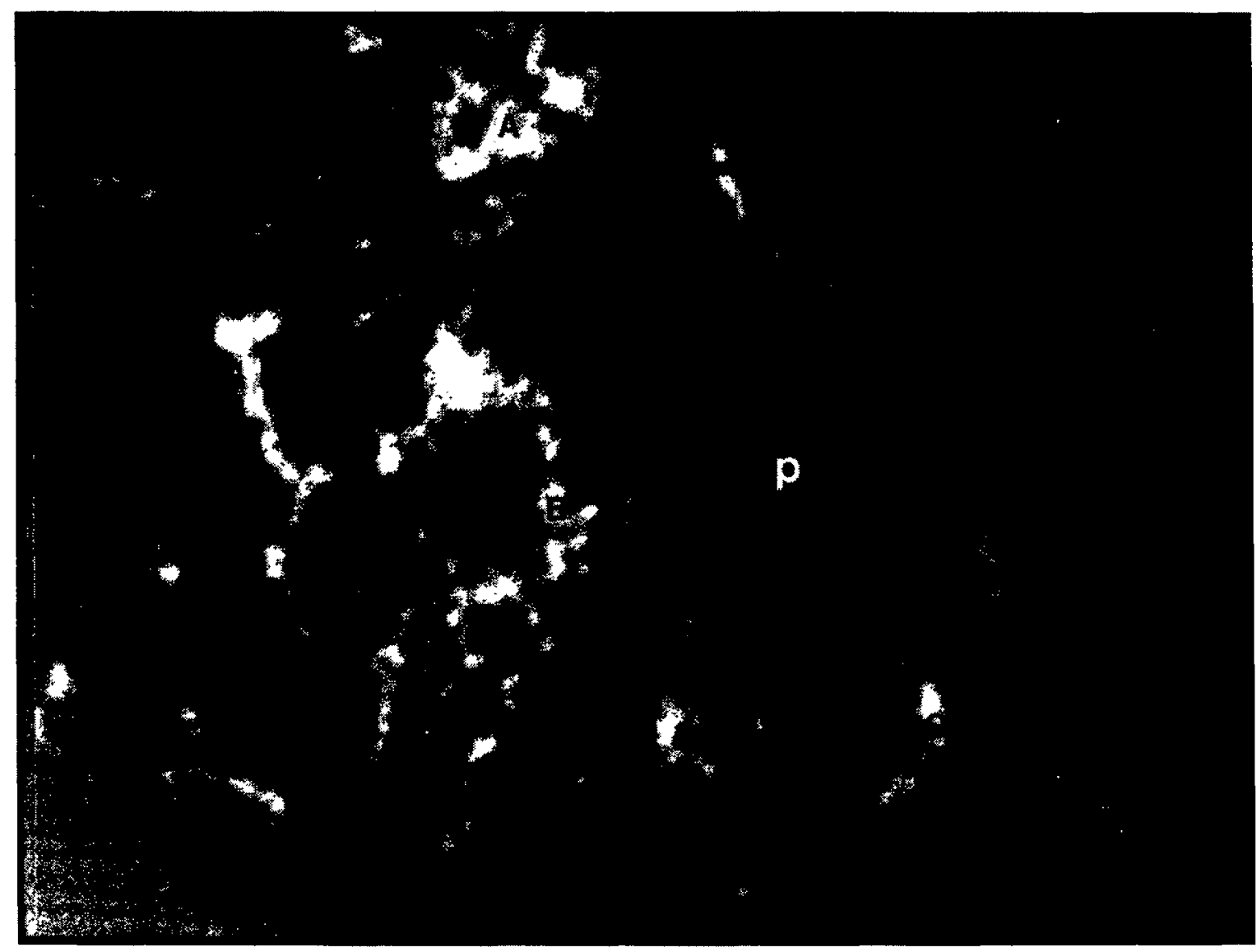

Fig. 6b.

\section{Little Regions}

Beside the active regions, the $\mathrm{X}$-ray image reveals a host of little bright regions which are largely the counterparts of the famous 'X-ray bright points' (XBPs). These regions are usually identified on X-ray images as small, isolated areas of emission, well separated from any surrounding structures (Golub et al., 1974). In fact, there has been no consistent definition, and no quantitative description in the case of counts. When counting stars or galaxies, one uses standard stars, limiting magnitudes, and a number of other calibrations. It is obvious that the number of stars counted depends on the exposure, seeing and other factors. Because such standards are not available, any detailed quantitative comparison, especially between different instruments in different wavelength bands, is arbitrary. The only useful comparison is by connection of the observed points with standard solar morphological phenomena.

Since there is no clear definition of what constitutes an XBP, we simply classify all isolated bright points smaller than 5 arc sec as such. We cannot at present place a brightness limit. However, we have also marked some ( $B P 1$ and 3$)$ that we believe would be counted in low resolution data. This definition already indicates a major new result from the NIXT observations: the XBPs in these data are smaller and 


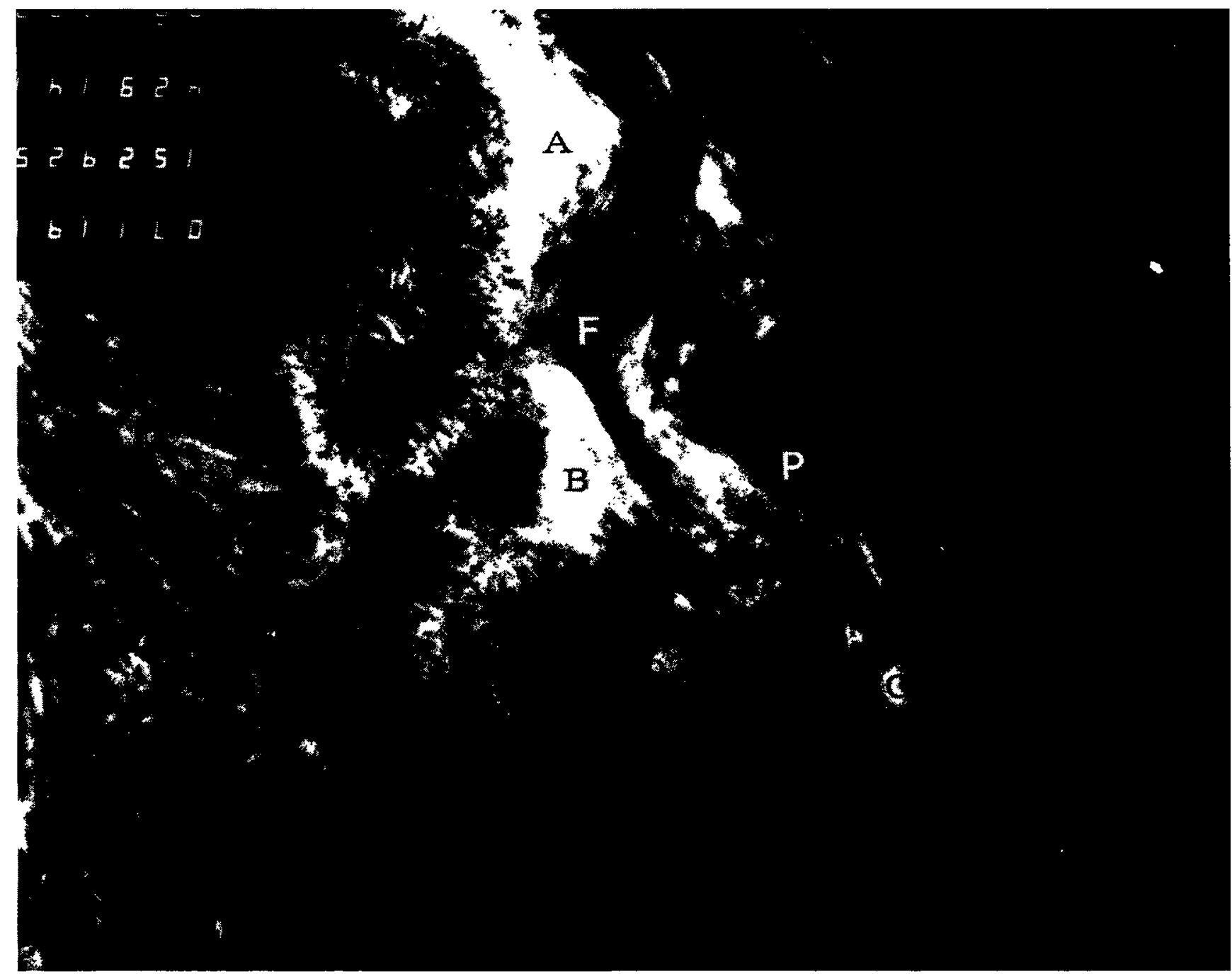

Fig. 6c.

more numerous than those in broadband grazing-incidence observations. There are at least two hundred such points on the solar disk. This may be due to the improved image quality of NIXT, or the improved contrast obtained by the ability of X-ray multilayers to isolate two spectral lines from the continuum. Certainly the first plays a role, since the points seen are considerably smaller than those shown by Webb et al. (1993), which were made with a lower-resolution Wolter telescope. Webb et al. give few details of their criteria for XBP other than agreement between the authors; we assume that they simply counted unresolved points. Dr Webb (private communication) informs us that their bright points are generally up to $30^{\prime \prime}$ in diameter; thus many are resolved in the NIXT frames.

For such regions, $B P 1$ through $B P 4$, are marked in Figures 1 and 2. Each is an example of resolved bipolar structure, visible from the BBSO $\mathrm{H} \alpha$ and the NSO full-disk magnetogram. While some are clearly resolved, all would likely appear as unresolved points in Webb et al. images as well as the earlier Skylab data. Other examples are the four small $\mathrm{X}$-ray regions situated in a $\mathrm{N}-\mathrm{S}$ band near disk 


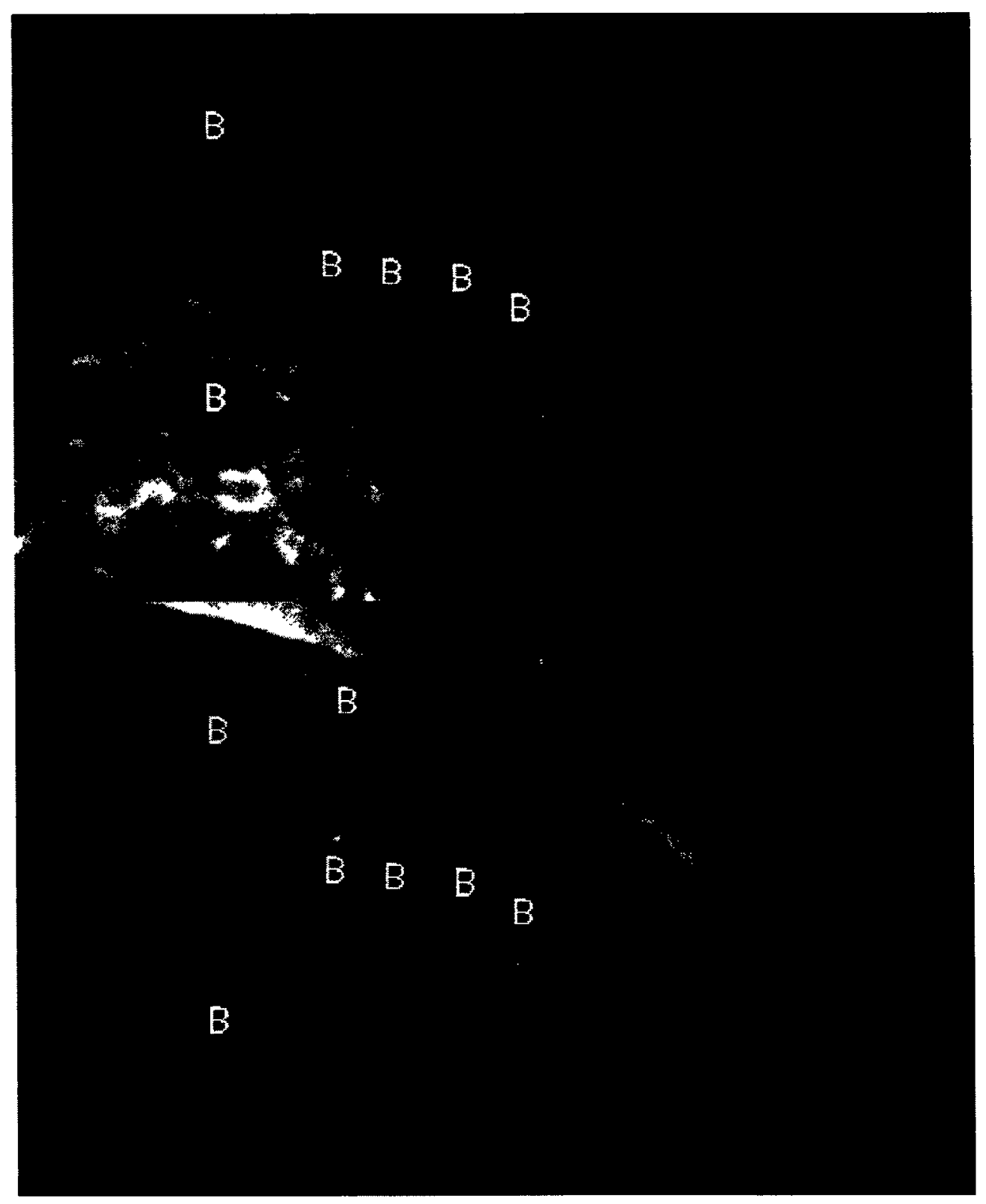

Fig. 7. Matching $\mathrm{H} \alpha$ (top) and $\mathrm{X}$-ray images of the SW quadrant. Because this was covered during the flight, a frame taken at 17:01 (before the eclipse) is used. Features corresponding to bipolar features on the NSO magnetogram are marked with a ' $B$ '.

center, in the vicinity of AR 6720. These regions are fairly long-lived, in that they are still visible in magnetograms taken on the next day; thus, they are borderline cases which might have been classified as small AR's in the Skylab analysis. Thus it is useless to count XBPs without a clear definition of limiting resolution and magnitude.

In Figure 7 we show an enlarged X-ray image of the SW quadrant with a corresponding $\mathrm{H} \alpha$ image taken after the eclipse. This quadrant was chosen because of the many $\mathrm{H} \alpha$ bright points near the limb, corresponding to an extended unipolar region. We have used the NSO magnetograms to mark the $\mathrm{H} \alpha$ and X-ray bright 
features corresponding to bipolar magnetic features with a ' $\mathrm{B}$ '. Identifications close to the limb are difficult. All of the brighter XBPs in the X-ray image have bright $\mathrm{H} \alpha$ counterparts, while the reverse is not true. However, all bipolar $\mathrm{H} \alpha$ features have corresponding bright X-ray emission, XBP if they are localized. With longer $\mathrm{X}$-ray exposure, those $\mathrm{H} \alpha$ features corresponding to unipolar field elements of the enhanced network mark the base of a diffuse coronal X-ray arch, the other end of which is not seen. These are the 'comet tails' seen on the disk and referred to above, and are particular clear near $B P 4$ (limitations of dynamic range make them difficult to see on the prints). This gives a consistent picture. The bipolar areas are bright and low because of heating and confinement. The stronger unipolar magnetic elements are the source of the bulk of the solar corona, at least the denser, brighter arches. The comet tails are formed by the coronal loops diverging from these features to connect to distant fields and trace out the field divergence. Presumaly the weaker $\mathrm{H} \alpha$ elements in the ordinary network also have such plumes but they are too weak to detect. Again, one requires a defined limiting magnitude.

It should be recalled that the radiation measured by NIXT comes from collisionally excited emission lines and is proportional to $n^{2}$, hence it exaggerates the contribution from the denser regions.

Webb et al. (1993) studied the time evolution of the magnetic features underlying the XBP and found that, while the bright points all corresponded to bipolar features, the majority were 'cancelling dipoles', a term used for magnetic elements of opposite sign which approach each other and disappear. Since it is physically impossible for magnetic fields to 'cancel', the fields involved must reconnect in such a way that they subside below the surface. An outward eruption after reconnection must leave behind a dipole to disappear by subsidence.

Unfortunately we do not have continuous magnetic coverage on the day of this flight, nor do we have detailed magnetograms, since we were carrying out eclipse programs. However, our full-disk $\mathrm{H} \alpha$ allows us to examine the fate of the $\mathrm{H} \alpha$ features identified with bright points in our data. While we could not follow every feature, none were seen to disappear in the course of the day, which suggests that they were not disappearing, but more likely stable. The only dependable indicator of X-ray brightening we can find is bipolarity. All of the brighter X-ray points for which we have magnetograms or adequate $\mathrm{H} \alpha$ are bipolar, we simply cannot say if there are growing, disappearing, or static. They are limb-brightened in X-rays. One other piece of information is the observation by Gary, Zirin, and Wang (1990) of the disappearance of such a dipole at $6 \mathrm{~cm}$ without any radio brightening. Thus these are not particularly energetic occurrences.

This result is consistent with an earlier one showing that regions which are either growing or shrinking on magnetograms look the same in X-rays (K. Harvey, 1992; Golub, Harvey, and Webb, 1986). This conclusion does not aply to EFRs, which are invariably brighter in X-rays while emerging. A reasonable extrapolation would suggest that emerging dipoles, rather than shrinking ones, are particularly bright. It is possible that the result of Webb et al. is due to the odd tendency for more 
elements to converge than diverge in the course of a day. We can understand this to be an observational effect because the density of magnetic elements is roughly constant with time.

We have noted our discovery of a new type of XBP, the satellite points in NOAAZ 6718. The resolution of grazing incidence $X$-ray imagers could not separate these from the active regions. At least six are visible in Figures 4 and 5, and some appear in the other regions. This shows another relation of X-ray brightness with the continuum of magnetic structures: the small bipolar XBPs must trap tightly; the long trajectories of the 'comet tails' can only cause low-level brightening, and the presence of a strong active source at the other end of a longer loop (even a sunspot) is sufficient to produce brightening in the satellite points. Whether the source is in the underlying $\mathrm{H} \alpha$ feature, which is invariably present, or in heat conveyed from the other end of the loop, cannot be determined.

\section{Filaments}

These are known to have an anti-correlation with the corona, associated with their location on horizontal fields, where there is no atmospheric heating. This distinct lack of X-ray emission occurs at the location of all our $\mathrm{H} \alpha$ filaments. It is therefore hard to decide if we are seeing absorption or just the absence of emission. Typically, X-ray images have shown filament cavities, indicating an absence of emitting material: several examples of this are seen in comparing Figures 1 and 2, such as the cavity associated with the filament at region 6720 , or the filament ' $F$ ', a large polar crown cavity.

In a few cases, filaments break a linear structure behind them, or otherwise display evidence of absorption. These cases are distinguished from the cavities by their sharp contours which coincide precisely with the locations of the corresponding $\mathrm{H} \alpha$ filament material. We discussed above the lower limits this places on the line-of-sight neutral atoms; the total number of atoms depends on the ionization. At the extreme left of Figure 2, between region 6722 and the limb, we see a filament which has some connection to a bright emission cloud at the limb. Part of the filament reaches the limb and obscures the X-ray emission behind it. There is some weak X-ray emission near the filament, but the filament itself is clearly dark and very sharply defined. This indicates that, in this case at least, we are seeing absorption rather than a coronal 'cavity' around the prominence.

The direction of the 'comets', which are probably the footpoints of long loops, is a clue to the three-dimensional field line configuration in their neighborhood. This may be seen nicely for filament number 9, across which a number of 'comets' in the X-ray image are seen to be directed from the NE. In this case, the comets turn to the right, which suggests magnetic fields trailing the poleward $\mathrm{H} \alpha$ component. They are also directed generally opposite the $\mathrm{H} \alpha$ fibril structure in the filament. The apparently contradictory field line directions inferred by these two methods suggest that filaments are more complicated than we have hitherto thought. 


\section{Summary}

We find a logical association between the magnetic and chromospheric structure underlying the corona and the coronal structure as evidenced by the NIXT frames. The brightest feature is the remains of the small flare. Filaments and sunspots are dark; regions of emerging flux or bipolar structure are bright. Every small plage or network element appears bright, mainly in $\mathrm{Mg} \mathrm{X}$. Plages with amorphous $\mathrm{H} \alpha$ structure are brighter in X-rays than those that are granular. Large-scale loops connect plage regions and EFRs. An EFR was observed to have a coronal condensation about twice the height of the corresponding $\mathrm{H} \alpha$ arches. Sunspots are dark and loops originating in them are also dark or faint. A preflare configuration was found with a bright X-ray loop crossing over a filament that erupted a few hours later.

Filaments are opaque, even in their short axis, giving a line-of-sight density of at least $4 \times 10^{20}$ neutral $\mathrm{H}$ atoms or $10^{19} \mathrm{He}$ atoms. These are large numbers, and suggest that the prominence gas is largely neutral. Objects identified as X-ray bright points show complex resolved structure; but a new class of bright points connected with satellite polarity was smaller and more complex. Essentially all vertical magnetic elements are bright in X-rays. Those connected to an opposite pole by a short loop are brighter than those connected over long paths. Isolated dipoles form a continuum; $\mathrm{X}$-ray bright points if they are small, active regions if they are large. Comet-tail structures projecting from unipolar network elements with a spread of less than $30 \mathrm{deg}$ are identified with spicule canopies, suggesting that fields do not diverge strongly from network elements.

\section{Acknowledgements}

The NIXT sounding rocket program is supported by NASA Grant NAG5-626 to the Smithsonian Institution (SI). Support for this collaborative program was provided by grant ATM-9119514 from the NSF to SI. The BBSO programs were supported by ONR grant N00014-89-J-1069, NASA NAGW-1972 and NSF ATM9122023, and part of Haimin Wang's participation by NASA GRO Fellowship NAG5-2090.

\section{References}

Allen, C. W.: 1981, Astrophysical Quantities, 3rd ed., Athlone Press, London, p. 98.

Chou, D. Y. and Zirin, H.: 1988, Astrophys. J. 333, 420.

Gary, D. E., Zirin, H., and Wang, H.: 1990, Astrophys. J. 355, 321.

Gaizauskas, V.: 1992, private communication.

Giovanelli, R. G. and Jones, H. P.: 1982, Solar Phys. 79, 267.

Golub, L. and Herant, M.: 1989, Proc. SPIE 1160.

Golub, L., Harvey, K. L., and Webb, D. F.: 1986, in A. I. Poland (ed.), NASA CP-2442.

Golub, L., Herant, M., Kalatka, K., and Lovas, I.: 1990, Nature 344, 842.

Harvey, J. W., Krieger, A. S., Timothy, A. F., and Vaiana, G. S.: 1975, in G. Righini (ed.), Oss. e Mem. Oss. Arcetri 104, 53.

Harvey, K.: 1992, personal communication. 
Peres, G., Reale, F., and Golub, L.: 1991, Proc. OSL Workshop, Capri.

Schmieder, B., Mein, N., Golub, L., Davila, J. M., Brosius, J., and Thomas, R.: 1992, in A. Poland and J. Mariska (eds.), Proc. SOHO Workshop, Annapolis MD.

Underwood, J. H., Bruner, M. E., Haisch, B. M., Brown, W., Acton, L. W.: 1987, Science 238, 61.

Vaiana, G. S., Krieger, A., and Timothy, A. F.: 1973, Solar Phys. 32, 81.

Vaiana, G. S. et al.: 1975, in G. Righini (ed.), Oss. e Mem. Oss. Arcetri 104.

Varsik, J.: 1994, Solar Phys., submitted.

Walker, A. B. C., Barbee, T. W., Jr., Hoover, R. B., and Lindblom, J. F.: 1988, Science 241, 1781.

Webb, D. F. and Zirin, H.: 1981, Solar Phys. 69, 99.

Webb, D. F., Martin, S. F., Moses, D., and Harvey, J. W.: 1993, Solar Phys. 144, 15. 